\title{
Comprehensive analysis of tornado statistics in comparison to earthquakes: intensity and temporal behaviour
}

\author{
L. Schielicke and P. Névir \\ Freie Universität Berlin, Institut für Meteorologie, Carl-Heinrich-Becker-Weg 6-10, 12165 Berlin, Germany \\ Correspondence to: L. Schielicke (lisa.schielicke@met.fu-berlin.de)
}

Received: 29 June 2012 - Revised: 13 December 2012 - Accepted: 17 December 2012 - Published: 15 January 2013

\begin{abstract}
Tornadoes and earthquakes are characterised by a high variability in their properties concerning intensity, geometric properties and temporal behaviour. Earthquakes are known for power-law behaviour in their intensity (Gutenberg-Richter law) and temporal statistics (e.g. Omori law and interevent waiting times). The observed similarity of high variability of these two phenomena motivated us to compare the statistical behaviour of tornadoes using seismological methods and quest for power-law behaviour. In general, the statistics of tornadoes show power-law behaviour partly coextensive with characteristic scales when the temporal resolution is high (10 to $60 \mathrm{~min}$ ). These characteristic scales match with the typical diurnal behaviour of tornadoes, which is characterised by a maximum of tornado occurrences in the late afternoon hours. Furthermore, the distributions support the observation that tornadoes cluster in time. Finally, we shortly discuss a possible similar underlying structure composed of heterogeneous, coupled, interactive threshold oscillators that possibly explains the observed behaviour.
\end{abstract}

\section{Introduction}

Tornadoes are not only characterised by violent wind speeds of a wide range of intensities, they are also highly variable in their lifetime (minutes up to hours), their path length (a few meters up to tens or hundreds of kilometres), and path width (meters up to several kilometres) (e.g. Brooks, 2004; Malamud and Turcotte, 2012). Additionally, tornadoes are observed to cluster in time (e.g. Brooks et al., 2003). Earthquakes are also observed to cluster in space and time and their properties and temporal behaviours are highly variable (e.g. Hainzl et al., 2003). A high variability in the properties of a system can be an indicator for fat tail distributions with power-law decay in the tail of the distribution.

Power-law or scale-free behaviour is often observed in complex systems whose behaviours arise from the nonlinear (multiplicative) interaction of a great number of degrees of freedom (e.g. Chakraborti and Patriarca, 2009; Love and Burton, 2005; Stanley et al., 2000). Especially in the case of power-law probability distributions, like that of Pareto, the scale-free behaviour can be derived from the assumption of a maximum Shannon entropy under the constraint of a constant geometric mean (e.g. Englehardt, 2002). This is in sharp contrast to an exponential behaviour of a probability function which is related to the constraint of a constant arithmetic mean. Moreover, it is assumed that systems characterised by power laws have a common underlying structure and that these systems are composed of interacting (coupled) nonlinear relaxational threshold oscillators (Osorio et al., 2010). In contrast to a normal oscillator, where charging and discharging of accumulated energy have the same frequency, a relaxational threshold oscillator is characterised by a relatively long accumulation period (also called charging or loading period) due to some external force and a sudden release of accumulated energy if a certain threshold is exceeded. This activity may in turn stimulate neighbouring elements. A threshold oscillator has two subsequent characteristic timescales, a timescale of loading and one of discharging, which are separated by one or more orders in time, e.g. for earthquakes the accumulation of stress can take periods up to several years, while the release process (the earthquake event) only takes seconds to minutes. Besides earthquakes, other examples of systems of coupled threshold oscillators are avalanches, forest fires, colonies of flashing fireflies (e.g. Herz and Hopfield, 1995), and seizures (Osorio et al., 2010). In the coupled atmosphere-ocean system, this 
principle is also used to describe the El Nino/Southern Oscillation (ENSO) phenomenon with the help of a delayed action oscillator (Suarez and Schopf, 1988). Systems with coupled heterogeneous threshold oscillators can exhibit different collective behaviour from totally (global) synchronised behaviour to self-organised criticality (SOC) characterised by power laws, or the coexistence of SOC and synchronization to totally incoherent behaviour (e.g. Herz and Hopfield, 1995; Osorio et al., 2010). The different observed regimes depend on the coupling strength and the heterogeneity of the system, where strong coupling leads to more synchronised behaviour, while large heterogeneity is more connected with self-organised criticality and power-law behaviour (Osorio et al., 2010). Osorio et al. (2010) compared five statistics ${ }^{1}$ of epileptic seizures and earthquakes concerning the event sizes and their temporal behaviour. Despite the differences in scales and constituent matters, Osorio et al. (2010) found power-law (scale-free) behaviour in the five statistics supporting a dynamical analogy between seizures and earthquakes. Differences were only observed in the precise values of the power-law exponents.

Earthquakes and tornadoes also show some analogies in their behaviours: for example, tornado intensity distributions in wind speed $v$ or Fujita intensity scale $F^{2}$ show similar behaviour for different regions in the world (e.g. Kurgansky, 2000; Brooks and Doswell, 2001; Dotzek et al., 2003, 2005; Feuerstein et al., 2005). Differences in the slopes of the distributions can be attributed to the dominant origin of the tornadoes in supercell- or non-supercell-dominated regions (Brooks and Doswell, 2001). Furthermore, if tornado magnitudes are derived in an analogous way to the seismic moments of earthquakes, the probability density distribution follows a power law with an exponent of comparable order to that of the Gutenberg-Richter law (Schielicke and Névir, 2011) which describes the relation between earthquake magnitudes and the number of earthquakes (Gutenberg and Richter, 1956). Similarly, slight regional variations in the power-law exponents of the Gutenberg-Richter law of earthquakes are observed (e.g. Ben-Zion, 2003; Godano and Pingue, 2000; Okal and Romanowicz, 1994). The variations depend on the investigated zone with smaller exponents in subduction zones than in mid-ocean ridge zones (e.g. Pisarenko and Sornette, 2003, 2004). Another indicator of analogous behaviour is the spatio-temporal clustering observed in tornado outbreaks (e.g. Doswell et al., 2006), even though the temporal behaviour of tornadoes has not been proved with respect to power-law behaviour so far. Likewise, earth-

\footnotetext{
${ }^{1}$ The five statistics are (1) the Gutenberg-Richter law of event sizes, (2) the distribution of time intervals between successive events, (3) the conditional waiting time until the next event, the (4) Omori and (5) inverse Omori laws.

${ }^{2}$ Tornadoes are rated by the maximum damage that occurred anywhere in their path. The rating is given in categories from $F 0$ (light damage) to $F 5$ (devastating damage) after Fujita (1971).
}

quakes are observed to cluster in space and time where temporal clustering implies that the distribution has a memory (Sornette and Knopoff, 1997). This can improve the estimate of the probability of a future earthquake by the knowledge of the times of previous ones (Sornette and Knopoff, 1997). Davis et al. (1989) showed that the observation "the longer it has been since the last earthquake, the longer the expected time till the next" is true for heavy-tailed distributions. Sornette and Knopoff (1997) expressed this property by the relation of the elapsed time since the last event to the conditional probability of occurrence of the next event. They showed that power law distributions are characterised by an increase of the expected time until the next event with increasing time elapsed since the last one. The temporal clustering of earthquakes can also be seen by the Omori law (e.g. Hainzl et al., 2003) which represents the increase in seismic activity after the occurrence of large earthquakes, so-called mainshocks (Omori, 1984). It follows a power law.

In conclusion, there are already some hints indicating analogies between earthquakes and tornadoes. While earthquakes are well studied concerning their magnitudes and their temporal behaviour, such a detailed analysis of tornadoes as well as a comprehensive comparison between both phenomena are still lacking. Motivated by the already observed power-law behaviour of tornado magnitudes analogously to the Gutenberg-Richter law of earthquake sizes (Schielicke and Névir, 2011) and by the work of Osorio et al. (2010), we will focus on the study of the temporal behaviour of tornadoes in comparison to earthquakes in this publication. Summarised, the aims of this work are to (1) study the tornado behaviour in time and intensity by applying seismological methods (following the example of Osorio et al., 2010) to tornado data and proof for power law or non-power-law behaviour; (2) to compare and discuss the tornado results with that of earthquakes; and (3) to discuss if the concept of heterogeneous, coupled, interactive threshold oscillators is in principle possible for tornadoes.

The presented paper is organised as follows: the data and inconsistencies in the tornado data base are described in Sect. 2. The methods for the analysis of the five-scale free distributions are explained in Sect. 3, followed by the results in Sect. 4. A conclusion and discussion of the results is given in Sect. 5.

\section{Data}

The earthquake data was provided by the Southern California Seismic Network (SCSN) catalogue. The network covers a region of about 32 to $37^{\circ} \mathrm{N}$ and -114 to $-121^{\circ} \mathrm{W}$ at its longest extent (Southern California region, for details see Hutton et al., 2010). The time period was chosen from 1981 to 2000 because of the consistency in network density and data processing. For the analysis, magnitudes to calculate seismic moments were used as well as the occurrence 
times of the earthquakes. The reported magnitudes are given by the local magnitude $M_{\mathrm{L}}$ up to a magnitude of 5, and for larger magnitudes $(M \geq 5)$ by the moment magnitude $M_{0}$, respectively. The spatial distribution of earthquakes was not considered. This might lead to slightly different results compared to published literature where the spatio-temporal clustering is of interest.

US Tornado data of the Storm Prediction Center (SPC, 2012) of NOAA (National Oceanic and Atmospheric Administration) has been used. The data set is available online under www.spc.noaa.gov. The Fujita classification, the path length and path width, and the time of occurrence of the tornadoes were used in the study. Unrated tornadoes or tornadoes with missing data of path length and width were only used in the temporal analyses of our work. Details are given in the single methods chapters. The data cover a period of $57 \mathrm{yr}$ from 1950 to 2006, which is identical to the Fujitascale rating era. Tornadoes observed before the implementation of the Fujita intensity scale in the mid-1970s were rated later retrospectively. This led to temporal inconsistencies in the data. Observed trends and possible reasons are described in more detail in, e.g. Schaefer and Edwards (1999), Verbout et al. (2006), and Doswell et al. (2009). An example is the averaged increase of the total yearly number of tornadoes by about 15 events per year from about 600 tornadoes per year in 1950 to about 1200 tornadoes in the 2000s (e.g. Verbout et al., 2006) mainly due to new weather observation technologies, changes in society, and enhanced communication. The increase of tornado reports concerns mainly weak tornadoes $(F 0, F 1)$, while there is a marked decrease of the number of tornadoes of Fujita scale $F 2$ and higher. Schaefer and Edwards (1999) presumed that this is most likely a result of the different ways tornadoes have been rated prior to the introduction of the Fujita-intensity scale, but they also point out that there is a high variability of tornado activity due to a natural year-to-year variance in the synoptic scale flow. Doswell et al. (2009) describe in detail the inconsistencies in the tornado data set due to changes in the rating practice. Additionally, Brooks (2004) found partly temporal instationarity of tornado path lengths and widths in the USA data set. He also reported that the policy of width reporting changed: prior to 1994 (including 1994) the mean width has been reported, after 1994 the maximum width has been reported, but the data appeared to show changes earlier than 1994.

\section{Methods}

In the following subsections the seismology based methods for obtaining the five statistics: Gutenberg-Richter law, interevent waiting times, expected time until the next event, Omori and inverse Omori-like behaviour are described in detail.

\subsection{Gutenberg-Richter law}

The Gutenberg-Richter law is one of the most famous, wellestablished empirical laws in seismology. It describes the relation between the magnitude $m$ of earthquakes and the number of earthquakes $N(>m)$ with a magnitude larger than $m$. If the magnitude is expressed by seismic moments $M_{0}$, the cumulative distribution equals a power law $(N(>$ $\left.M_{0}\right) \sim M_{0}^{-\beta}$ ). The corresponding probability density distribution is then a power law with the exponent diminished by $1\left(n\left(M_{0}\right) \sim M_{0}^{-\beta-1}\right.$ ), where $\beta=2 / 3 b$ with $b \approx 1$ (e.g. BenZion, 2003).

\subsubsection{Calculation of the seismic moments of earthquakes}

The seismic moment of earthquakes is a measure for the size of an earthquake and is related to the energy released during the rupture process. The seismic moment represents a combination of the geometrical properties of the fault with the static stress drop, which is given by the difference between initial and final stress values before and after the earthquake (BenZion, 2003). The seismic moment $M_{0}$ is connected with the moment magnitude $M_{\mathrm{w}}$ by (after Hutton et al., 2010):

$\log _{10}\left(M_{0}\right)=1.5 \cdot M_{\mathrm{w}}+16.095$,

where $\beta^{-1}=1.5$ is the exponent of the Gutenberg-Richter law and $M_{0}=10^{16.095} \mathrm{Nm}$ represents the seismic moment of an earthquake with zero magnitude $\left(M_{\mathrm{w}}=0\right)$. This formula was used to calculate the seismic moments of earthquakes with magnitudes $M \geq 5$. For earthquakes with smaller magnitudes $(M<5)$ the SCSN catalogue gives the local magnitudes $M_{\mathrm{L}}$ of earthquakes. In this case seismic moments $M_{0}$ are calculated by the following expression:

$\log _{10}\left(M_{0}\right)=1.17 \cdot M_{\mathrm{L}}+17.32$.

This formula was derived by a regression fit to the data in the SCSN catalogue (after Hutton et al., 2010) and gives the relation of the seismic moment scale to the local magnitude scale.

\subsubsection{Calculation of atmospheric moments of tornadoes}

The atmospheric moment was designed analogously to the seismic moment and represents an estimate for the total work that was necessary for the generation of the tornado. In the case of tornadoes, the atmospheric moment $M_{\mathrm{a}}$ is estimated by (Schielicke and Névir, 2011):

$M_{\mathrm{a}} \approx A L \bar{\rho} e$,

where $A$ is the circular area of the tornado on the ground ( $A=\pi d^{2} / 4, d$ : diameter), $L$ is the path length of the tornado, $\bar{\rho}$ is the average density which is assumed to be equal 
to $1 \mathrm{~kg} \mathrm{~m}^{-3}$, and $e$ is the mass-specific kinetic energy (see also Schielicke and Névir, 2009) which is given by

$e=\langle v(F)\rangle^{2} / 2$.

The mean value of the wind speed concerning a specific Fujita class, $\langle v(F)\rangle$, is calculated by the relation of Fujita intensity scale and velocity, where $\langle v(F)\rangle=6.30 \mathrm{~m} \mathrm{~s}^{-1}(F+$ $2.5)^{3 / 2}$. Atmospheric moments have only been calculated for tornadoes with non-zero path width and length reports as well as a rating between $F 0$ and $F 5$. This applied to 47962 of 50403 tornadoes reported in the period from 1950 to 2006. The tornado numbers concerning their Fujita classification are listed in Table 1.

\subsection{Interevent waiting times}

Each event (earthquake/tornado) has a documented time of occurrence in its report. The interevent waiting time between two successive events has been calculated by the difference of their times of occurrence. Probability density estimates of these interevent waiting times has been calculated with a histogram-based method with different bin sizes: equally spaced bins of $10 \mathrm{~min}, 60 \mathrm{~min}$, and $1440 \mathrm{~min}$ (=1 day) and logarithmically spaced bins with increasing bins sizes of $10^{-1+0.2 i}$ min with $i \in[0,1, \ldots, 36]$. Linear fits have been applied to the double-logarithmically plotted data. In general, the shorter timescales up to $600 \mathrm{~min}$ was analysed with histograms based on 10 and $60 \mathrm{~min}$ bins, while the long timescale larger than one day was analysed with histograms based on $1440 \mathrm{~min}$ (equal to 1 day) bins. Note that the very short timescale up to $60 \mathrm{~min}$ can be associated with the convective scale, the short timescale ranging from 60 up to about $600 \mathrm{~min}$ with the mesoscale and the long timescale with the synoptic scale. The histograms based on logarithmically spaced bins were used to analyse data in the whole time range. This was applied to the total data set and to tornadoes with Fujita intensities larger than and equal to $F 2$ (hereafter called $F 2+$ tornadoes) as well as to earthquakes with magnitudes $M \geq 3$. In the latter two cases, the data sets were reduced to events with these moments/Fujita scales and the probability density estimates of the interevent waiting times have been calculated only in these reduced data sets. Additionally, we analysed the monthly variations of the interevent waiting times by concatenating the interevent waiting times of the single months (e.g. only Januaries) over the whole period (1950-2006) for all tornadoes. For the analysis in the mesoscale and synoptic scale, we used the histogram-based method described above applied on the resulting monthly data sets.

\subsection{Expected time until the next event}

The following derivation of the expected time until the next event is adopted from Sornette and Knopoff (1997). Assume that $p(t)$ is the probability density of the time intervals
Table 1. Tornado numbers in Fujita intensity classes from $F 0$ to $F 5$ with F-9 indicating unrated tornadoes, source: SPC, period: 1950 2006.

\begin{tabular}{lcccccccc}
\hline Period & $F-9$ & $F 0$ & $F 1$ & $F 2$ & $F 3$ & $F 4$ & $F 5$ & total \\
\hline $1950-1959$ & 504 & 767 & 1660 & 1381 & 364 & 108 & 12 & 4796 \\
$1960-1969$ & 778 & 1370 & 2257 & 1859 & 439 & 99 & 11 & 6813 \\
$1970-1979$ & 557 & 2178 & 3416 & 1832 & 474 & 108 & 14 & 8579 \\
$1980-1989$ & 25 & 3278 & 3307 & 1211 & 308 & 64 & 3 & 8196 \\
$1990-1999$ & 0 & 7371 & 3272 & 1063 & 338 & 82 & 10 & 12136 \\
$2000-2006$ & 0 & 6438 & 2521 & 690 & 195 & 34 & 1 & 9879 \\
\hline $1950-2006$ & 1864 & 21402 & 16433 & 8036 & 2118 & 495 & 51 & 50399
\end{tabular}

between two successive events (In our analysis, this probability density is given by the histogram-based pdf estimates of the interevent waiting times between earthquakes/tornadoes); if $t$ is the time since the last event, what is the probability density function $P\left(t^{\prime}\right)$ that the additional waiting time until the next event is $t^{\prime}$ ? This is given by a conditional probability (after Sornette and Knopoff, 1997, their Eq. 2):

$P\left(t^{\prime}\right)=\frac{p\left(t+t^{\prime}\right)}{\int_{t}^{\infty} p(s) \mathrm{d} s}$,

where $p\left(t+t^{\prime}\right)$ gives the probability that the next event will occur at time $t^{\prime}$ from $t$ on and $\int_{t}^{\infty} p(s) \mathrm{d} s$ is the probability that no earthquake or tornado event has occurred up to time $t$ ( $s$ denotes the time variable used for the integration). The average expected time until the next event, expressed by $\left\langle t^{\prime}\right\rangle$, is calculated as a function of the time since the last event and is given by (after Sornette and Knopoff, 1997, their Eq. 3):

$\left\langle t^{\prime}\right\rangle=\frac{\int_{0}^{\infty} t^{\prime} p\left(t+t^{\prime}\right) \mathrm{d} t^{\prime}}{\int_{t}^{\infty} p(s) \mathrm{d} s}$

The denominator is the first cumulative integral of the probability density function $p(t)$ and the numerator is the second cumulative integral of $p(t)$. If $p(t)=f^{\prime \prime}(t)$, the averaged expected time to the next earthquake (tornado) can be expressed by (after Sornette and Knopoff, 1997, their Eq. 6):

$\left\langle t^{\prime}\right\rangle=-\frac{f(t)}{f^{\prime}(t)}$.

The pdf estimates of the earthquake/tornado interevent waiting times have been identified in the previous chapter and were used to calculate the first and second cumulative integral with help of Eq. (1). The average unconditional in terevent waiting time $\langle t\rangle$ was calculated by (after Sornette and Knopoff, 1997):

$\langle t\rangle=\int_{0}^{\infty} t p(t) \mathrm{d} t$. 
Table 2. Relative number of tornadoes with atmospheric moments $M \geq 10^{10} \mathrm{~J}$ relative to the observed tornadoes per Fujita class $(F 0-F 5)$. For unrated tornadoes $F-9$ no moments were calculated, source: SPC, period: 1950-2006.

\begin{tabular}{lccccccc}
\hline Period & $F-9$ & $F 0$ & $F 1$ & $F 2$ & $F 3$ & $F 4$ & $F 5$ \\
\hline $1950-1959$ & - & 0.067 & 0.235 & 0.488 & 0.758 & 0.972 & 0.917 \\
$1960-1969$ & - & 0.031 & 0.182 & 0.426 & 0.708 & 0.960 & 1.000 \\
$1970-1979$ & - & 0.025 & 0.221 & 0.522 & 0.816 & 0.981 & 1.000 \\
$1980-1989$ & - & 0.026 & 0.218 & 0.700 & 0.958 & 0.953 & 1.000 \\
$1990-1999$ & - & 0.028 & 0.373 & 0.828 & 0.968 & 0.988 & 1.000 \\
$2005-2006$ & - & 0.055 & 0.562 & 0.933 & 0.995 & 1.000 & 1.000 \\
\hline $1950-2006$ & - & 0.037 & 0.299 & 0.597 & 0.845 & 0.974 & 0.980 \\
\hline
\end{tabular}

\subsection{Omori and inverse Omori law}

The Omori law describes the increase of seismic activity followed after the occurrence of a large earthquake. This behaviour can be described by a power-law decay proportional to $\sim 1 /\left(t-t_{c}\right)^{p}$ with observed values of $p$ near 1 , where $t$ represents the time after the occurrence of the mainshock at time $t_{c}$ (e.g. Osorio et al., 2010). The analysis of the foreshock sequence is claimed to have similar behaviour $\sim 1 /\left(t_{c}-t\right)^{p^{\prime}}$ but with a different exponent $p^{\prime}$. It describes the increase in activity before a mainshock (so-called foreshocks). Foreshocks are not always observed, so that the inverse Omori law could only be observed by stacking the foreshock activity of a large ensemble of different mainshocks (e.g. Kagan and Knopoff, 1978; Jones and Molnar, 1979). Maeda (1999) analysed the $p^{\prime}$-value that describes the foreshock activity and observed it to be significantly smaller than the aftershock exponent $p$ when using a stacking method which only included one foreshock event and excluded swarm type activities. Excluding swarm type activity means that the aftershocks should be at least one order of magnitude smaller than the mainshock. But Maeda (1999) also remarked that the difference between the exponents becomes smaller and insignificant if the value for the aftershock exponent is calculated including swarm type activities. In this case the difference in magnitude is only required to be larger than zero between main- and aftershock.

In the case of tornadoes, we analysed the tornado activity around a main tornado event. These main events were defined as tornadoes with atmospheric moments of at least $10^{10} \mathrm{~J}$. This criterion applied to 12821 tornadoes, including almost all $F 4$ and $F 5$ events (see Table 2). A stacking method was used to calculate the tornado activity before and after a main event: stacking tornado activity means that we took the total sum of tornado moments that occurred on a certain time (in 1 min bins) around the main tornado. It should be remarked that the method allows a single event to be a preceding, a following and a main event if it is followed or preceded by another main event of at least $10^{10} \mathrm{~J}$. This is rather similar to the method including swarm-type activity of earthquakes
Table 3. Exponents of power law pdfs (estimates) of tornadoes and earthquakes. Abbreviations: $F 2+$ : tornadoes with Fujita intensities larger than or equal to $F 2$, all: tornadoes of all intensities, mesosc.: mesoscale (here: times between 60 and $600 \mathrm{~min}$ ), syn.sc.: synoptic scale (here: times larger than one day), $M$ : magnitude.

\begin{tabular}{llll}
\hline & Statistic & Tornadoes & Earthquakes \\
\hline 1. & Gutenberg-Richter law & -1.2 & -1.8 \\
& (Fig. 1) & & \\
2. & Interevent waiting times & -1.7 (all, mesosc.) & $-1.0($ for $M \geq 3)$ \\
& (Figs. 2 and 3a, b) & $-1.6(F 2+$, mesosc. $)$ & \\
& & $-2.1($ all, syn.sc. $)$ & \\
& & $-1.4(F 2+$, syn.sc. $)$ & \\
3. & Expected time until the & $+0.40($ all $)$ & $+0.15($ for $M \geq 2)$ \\
& next event (Fig. 4) & $+0.25(F 2+)$ & $+0.11($ for $M \geq 3)$ \\
4. & Omori law (Fig. 5) & -1.0 & -1.0 \\
5. & Inverse Omori law & -1.0 & $\approx-0.7 \ldots-1.0$ \\
& (Fig. 5) & & after Maeda $(1999)$ \\
\hline
\end{tabular}

(Maeda, 1999). The curve was normalised by summing up all moments in different bin sizes (for example $60 \mathrm{~min}$ ) divided by the bin size.

\section{Results}

Five statistics (Gutenberg-Richter law, interevent waiting times, expected time until the next event, Omori and inverse Omori law) have been used to compare tornadoes with earthquakes. The results are summarised in Table 3.

\subsection{Gutenberg-Richter law}

Figure 1 shows the probability density functions (pdf) of earthquakes and tornadoes concerning their seismic moments and atmospheric moments, respectively. Both pdfs show power-law behaviour with slightly different exponents -1.80 for earthquakes of the SCSN catalogue and -1.19 for tornadoes.

\subsection{Interevent waiting times}

The probability density estimates of the interevent waiting times of earthquakes with magnitudes $M \geq 3$, of all tornadoes and of tornadoes with Fujita intensities larger than or equal to $F 2$ (hereafter called $F 2+$ tornadoes) are presented in Fig. 2. Logarithmically spaced bin sizes in time were used. The exponent of earthquakes in the range of about 2.5 to $250 \mathrm{~min}$ is approximately -1 . For tornadoes, the following results are observed: on the shorter timescales (from about 60 to $1000 \mathrm{~min}$, mesoscale) the distributions of all and $F 2+$ tornadoes are nearly indistinguishable, while on the very short timescale (up to $60 \mathrm{~min}$, convective scale) as well as on larger timescales (larger than $1000 \mathrm{~min}$, synoptic scale) the distributions deviate with different slopes; a clear peak of the distributions occurs in the range of about $1000 \mathrm{~min}$, indicating a characteristic timescale. For a more detailed analysis of the tornado distributions in the different time regimes equally 


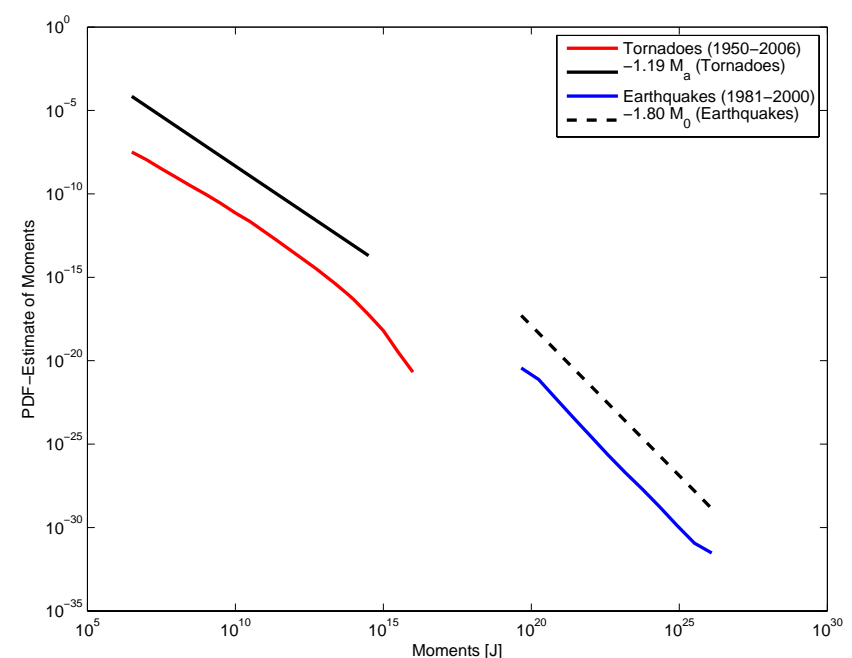

Fig. 1. Gutenberg-Richter law: Comparison of probability density distributions of tornadoes (1950-2006, USA, red curve) and earthquakes (1981-2000, SCSN catalogue, blue curve) concerning their moments: atmospheric moments $M_{\mathrm{a}}$ and seismic moments $M_{0}$. Linear fits have been applied to the double-logarithmic plot of the data (black solid line for tornadoes, black dashed line for earthquakes) with slopes of -1.19 for tornadoes and -1.80 for earthquakes.

spaced time bins were used. Figure 3 a shows the distributions based on $60 \mathrm{~min}$ bins. Clear peaks can be identified near $1440 \mathrm{~min}$ (=1 day) and multiples of the latter. Around the time of the first peak the distributions start to deviate increasingly with time. The fit of the distributions for times from 60 to $600 \mathrm{~min}$ show a power-law decay with nearly identical exponents of -1.67 and -1.60 for all tornadoes and for $F 2+$ tornadoes, respectively. Figure $3 \mathrm{~b}$ shows the probability density estimates of the interevent waiting times based on $1440 \mathrm{~min}$ bins. The distributions have power-law decay with different exponents: -2.1 for all tornadoes, and -1.35 for $F 2+$ tornadoes. This is in accordance with the findings of N. Dotzek (personal communication, 2005), who reported a power-law decay of about -2.2 for return times of US tornado days and of about -1.0 for return times of German tornado days. The characteristic scales around multiples of 1 day are only observed in the analysis based on bin sizes of 10 (not shown) and $60 \mathrm{~min}$, while the analysis based on the $24 \mathrm{~h}$ bin size smooths out the effect of characteristic scales. Additionally, we analysed the monthly variations for all tornadoes in the short (from 60 to $600 \mathrm{~min}$ ) and in the long time ranges (larger than 1 day) in Fig. 4. The exponents of the short time range (blue line) differ only slightly during the year, while the exponents of the long time range (red line) show a clear variation over the year with a negative peak or strongly negative exponents about -3.5 in summer (May to July) and higher negative values around -1 in winter (November to January).

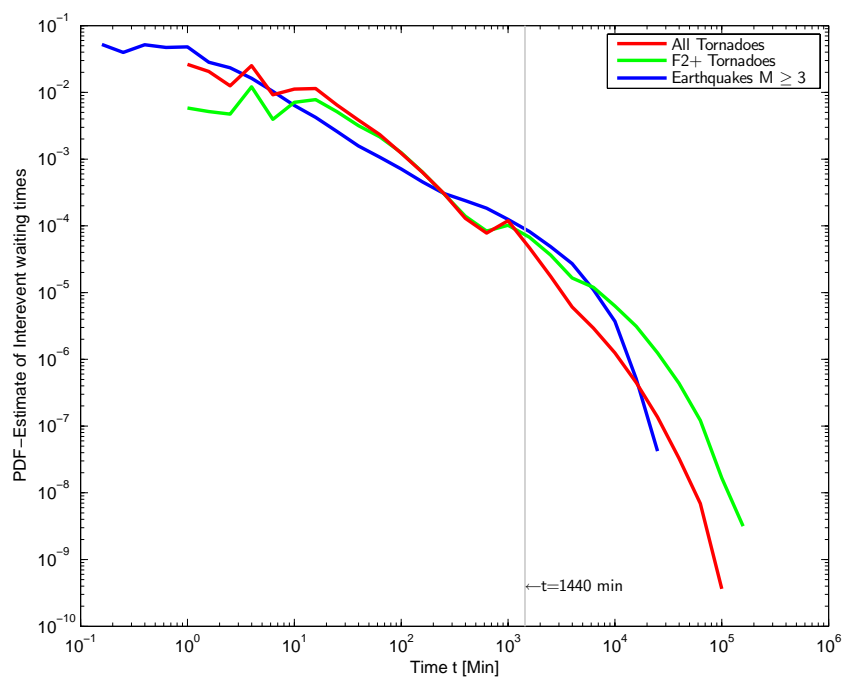

Fig. 2. Interevent waiting times: Comparison of the probability density distributions of earthquakes with magnitudes larger than or equal to 3 (1981-2000, SCSN catalogue, blue curve), all tornadoes (1950-2006, USA, red curve) and tornadoes with intensities larger than or equal to $F 2$ ( $F 2+$ tornadoes, 1950-2006, USA, green curve), based on logarithmically spaced time bins with increasing bin sizes of $10^{(-1+0.2 i)}$ min with $i \in[0,1, \ldots, 36]$. The time line of 1440 minutes (equal to 1 day) is highlighted by the grey vertical line in the plot.

Note that earthquakes and tornadoes have probability density distributions with heavy tails with exponents of $\beta<1$ (except for the fits in the long time ranges from April to August in Fig. 4). Therefore, a large range of observed interevent waiting times is observed such that mean and variance of the distributions are not well defined.

\subsection{Expected time until the next event}

The calculation of the average (unconditional) waiting time (Eq. 8) of tornadoes gives a value of about $600 \mathrm{~min}(10 \mathrm{~h}$ ) for the occurrence of a tornado in the United States. The mean waiting time for having a tornado of at least Fujita intensity $F 2$ is about 2800 min (approximately 2 days). However, it is rather observed that tornadoes cluster in time.

The average conditional waiting time (Eq. 6) for the tornado series including all and only events with at least $\mathrm{Fu}$ jita intensity scale $F 2(F 2+)$ is shown in Fig. 5. The distributions are approximated by power laws with a steeper slope for all tornadoes $(\approx 0.40)$ than for the $F 2+$ tornadoes $(\approx 0.25)$. A steeper slope is related with a faster increase of the expected time until the next event with increasing time elapsed since the last one. Otherwise the values for $F 2+$ tornadoes are higher for the same time elapsed since the last tornado for the whole range of the observed curves. In both cases, at about 10 to $20 \mathrm{~min}$ after the occurrence of the last tornado, the averaged expected, conditional waiting time exceeds the unconditional waiting time, such that the expected 


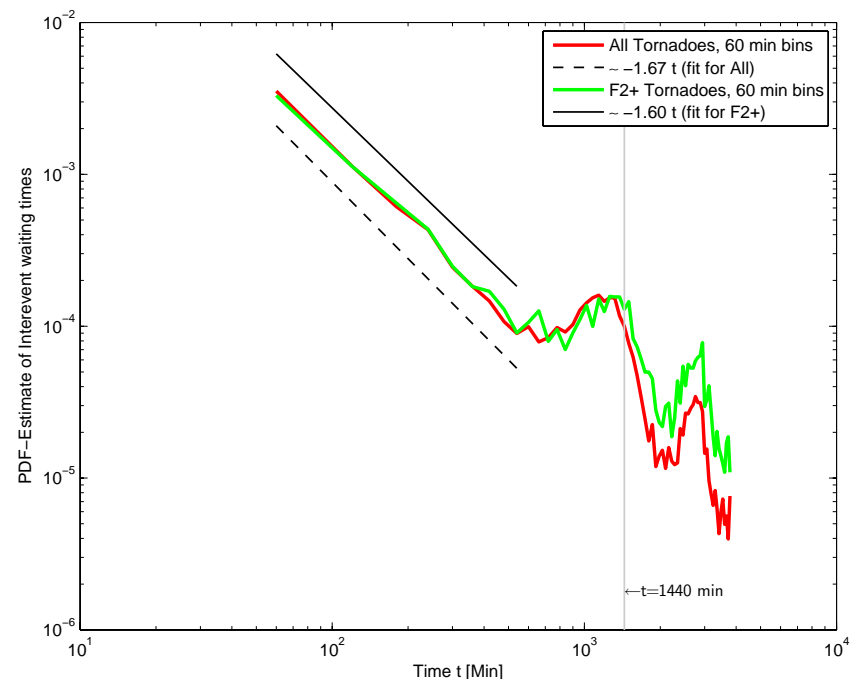

Fig. 3a. Short time analysis of interevent waiting times for all tornadoes (1950-2006, USA, red curve) and for tornadoes with Fujita intensities larger than or equal to $F 2$ ( $F 2+$ tornadoes, 1950-2006, USA, green curve), based on 60 min bins. Linear fits have been applied to the double-logarithmic plotted data in the range of 60 to 600 min with slopes of -1.67 for all tornadoes (black dashed line) and -1.60 for $F 2+$ tornadoes (black solid line). The time line of $1440 \mathrm{~min}$ (equal to 1 day) is highlighted by the grey vertical line in the plot.

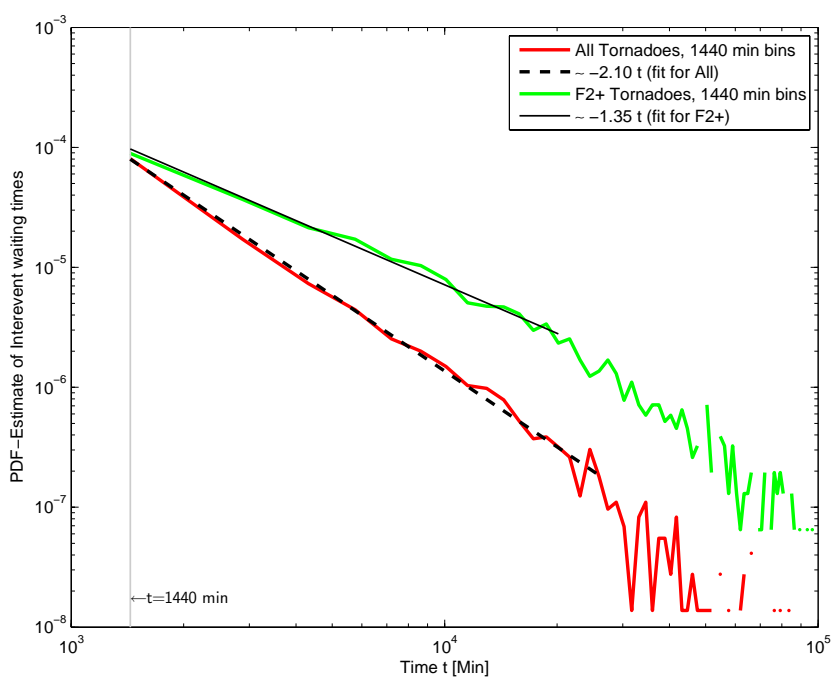

Fig. 3b. Long time analysis of interevent waiting times for all tornadoes (1950-2006, USA, red curve) and for tornadoes with Fujita intensities larger than or equal to $F 2(F 2+$ tornadoes, 1950 2006, USA, green curve), based on 1440 min bins. Linear fits have been applied to the double-logarithmic plotted data in the range of 1 day (1440 $\mathrm{min}$ ) to about 14 days (about $20000 \mathrm{~min}$ ) with slopes of -2.10 for all tornadoes (black dashed line) and -1.35 for $F 2+$ tornadoes (black solid line). The time line of $1440 \mathrm{~min}$ (equal to 1 day) is highlighted by the grey vertical line in the plot.

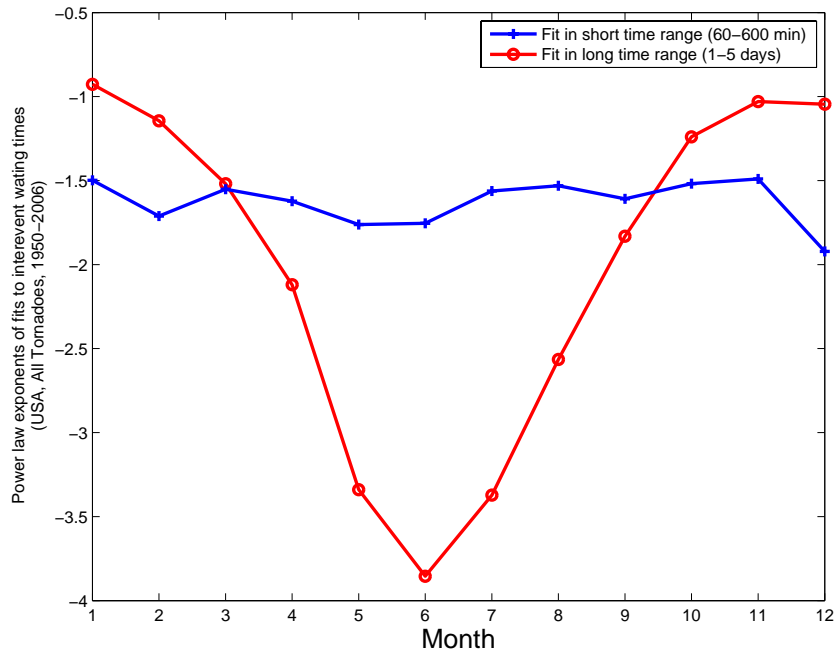

Fig. 4. Monthly analysis of the interevent waiting times of US tornadoes (1950-2006): Plotted are the exponents of the power law fits (= slopes of linear fits to double-logarithmic plotted data) in different time regimes: short time range up to $600 \mathrm{~min}$ (blue line, fit time range for every month: 60 to $600 \mathrm{~min}$, data in $60 \mathrm{~min}$ bins) and long time range starting with 1 day (red line, fit time range for every month: 1 to 5 days, data in 1440 min bins).

time until the next event increases with increasing elapsed times. On the other hand, the expected time until the next event is smaller than the averaged unconditional time before that point, theoretically up to 0 at $t=0$, directly after the occurrence of a tornado. This means that the probability for having a tornado in the near future is very high. It therefore describes the temporal clustering of tornadoes.

Expected times until the next event were also calculated for earthquakes with magnitudes $M \geq 2$ (light blue curve) and with magnitudes $M \geq 3$ (blue curve). These curves increase with time, but with different exponents than the tornadoes: 0.15 for $M \geq 2$ and 0.11 for $M \geq 3$. Likewise to the tornado curves, the increase of the curves including stronger events $(F 2+, M \geq 3)$ is smaller. However, the exponents of the earthquake curves differ only slightly.

\subsection{Omori-like and inverse Omori-like behaviour}

Figure 5 shows the tornado activity around main tornado events. Main tornadoes were defined by atmospheric moments larger equal $10^{10} \mathrm{~J}$. The normalised $1440 \mathrm{~min}(=24 \mathrm{~h})$ curves follow approximately power laws before and after the main event with a decay of -1 analogous to the Omori-law of earthquakes for the aftershock sequence. In contrast to the earthquakes where foreshock and aftershock sequence are antisymmetric (see e.g. Osorio et al., 2010), the increase in tornado activity is symmetric. The fastest decay is observed near the main event. 


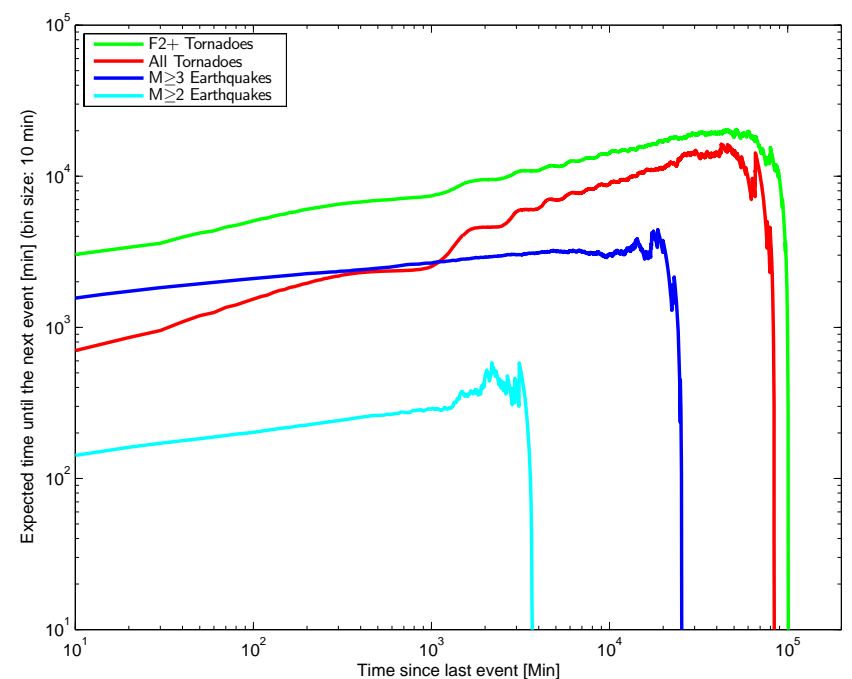

Fig. 5. Expected time until the next event: for all tornadoes (19502006, USA, red curve) and for tornadoes with Fujita intensities larger than or equal to $F 2$ ( $F 2+$ tornadoes, 1950-2006, USA, green curve), for earthquakes (1981-2000, SCSN catalogue) with magnitudes $M \geq 3$ (dark blue curve), and with magnitudes $M \geq 2$ (light blue curve). Data in 10 min bin sizes.

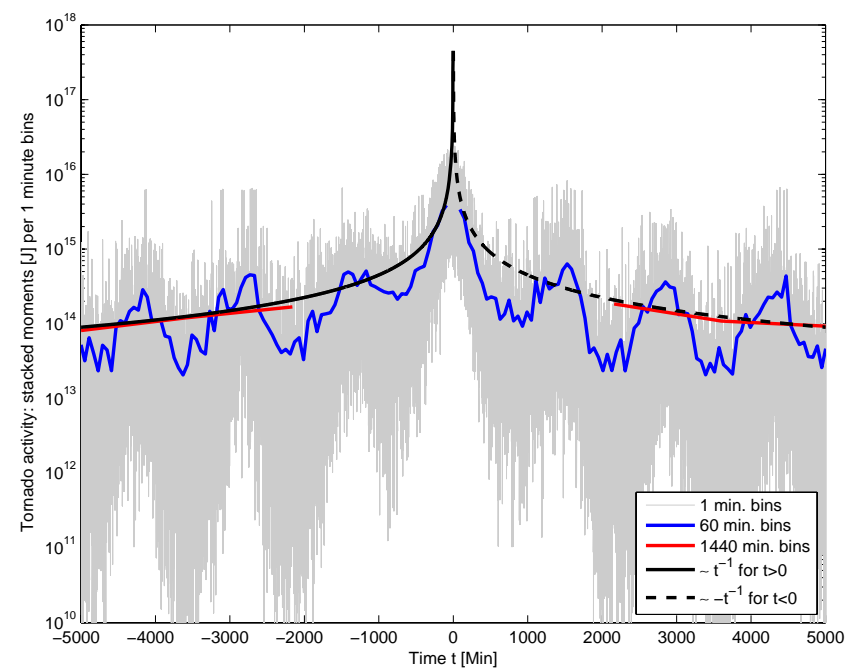

Fig. 6. Omori-like behaviour in tornado activity following and proceeding a tornado event with atmospheric moments of at least $M \geq 10^{10} \mathrm{~J}$, a stacking method of tornado moments is used (USA tornado data, 1950-2006). Grey, blue and red lines represent the $1 \mathrm{~min}, 60 \mathrm{~min}$ and $1440 \mathrm{~min}$ bins, respectively. Black solid and black dashed line are fitted to the fore- and aftershock sequences following power laws proportional to $f_{\text {fore }}(t) \sim-t^{-1}$ and $f_{\text {after }}(t) \sim t^{-1}$, respectively ( $t$ : time).
Table 4. Separation of time scales for different oscillators. Abbreviations: syn.sc.: synoptic scale, conv.sc.: convective scale.

\begin{tabular}{|c|c|c|c|}
\hline Oscillator & $\begin{array}{l}\text { Loading time } \\
L\end{array}$ & $\begin{array}{l}\text { Discharging time } \\
D\end{array}$ & $\begin{array}{l}\text { ratio of } \\
D / L\end{array}$ \\
\hline Harmonic & $L=D$ & $L=D$ & 1 \\
\hline ENSO & $4 \mathrm{yr}$ & $3-6$ months (season) & $10^{-1}$ \\
\hline Tornadoes & 3-7 days (syn.sc.) & $10 \min ($ conv.sc. $)$ & $10^{-3}$ \\
\hline Earthquakes & years & seconds - minutes & $10^{-8}$ \\
\hline
\end{tabular}

\subsection{Is the threshold oscillator concept applicable to tornadoes?}

Osorio et al. (2010) discussed the similarities of the underlying structures of earthquakes and seizures justifying, at first glance, a comparison between these very different phenomena. They argued that the events (earthquakes/seizures) supporting elements (fault networks/neuronal assemblies) are composed of interactive, coupled, heterogeneous threshold oscillators. In the case of the atmosphere, it can be speculated whether the concept of coupled, heterogeneous threshold oscillators is applicable as well. For severe convection, the favouring conditions or ingredients are accumulated on the large synoptic scale over a relatively large period, while the release processes (e.g. convection, tornadoes) only take a short period in time (seconds to minutes in the case of tornadoes). The coupling takes place in the atmosphere starting from neighbouring air parcels up to the interaction of synoptic, meso- and convective scale (the interaction between synoptic-scale processes and severe convection is described in Doswell and Bosart, 2001). The heterogeneity can for example be identified by different arrangements of favouring ingredients as well as different cap sizes or values of CIN (convective inhibition). That is equal to different threshold sizes. Other examples providing heterogeneity are local temperature or moisture gradients or different boundary conditions like orographic and surface effects. In conclusion, coupling and heterogeneity of the different arrangements of favouring conditions play an important role in the formation of tornadoes. In general, a threshold oscillator is characterised by two characteristic timescales of loading and discharging, which are separated by one or more orders in time. In Table 4, we summarised this oscillator typical parameter (a ratio of typical discharging and charging times) for different oscillator types: harmonic oscillator, delayed action oscillator describing the ENSO phenomenon, tornadoes and earthquakes. We used characteristic orders of the timescales of the phenomena. Earthquakes have the smallest parameter and the largest separation of timescales, respectively, followed by tornadoes and ENSO, while the harmonic oscillator has a parameter value of 1 identical to no separation of timescales. Concluding, the concept of threshold oscillators can in principle also be applied to tornadoes, but a detailed theory so far is wide beyond the scope of our paper. This would mean that 
tornadoes and earthquakes have a similar underlying structure. In that case, a comparison between both phenomena is justified.

\section{Discussion and conclusions}

In this paper, we analysed tornado behaviour on the basis of five statistics concerning different properties with focus on the temporal behaviour of tornadoes and compared the results with that of earthquakes. Therefore, we applied seismological methods to tornadoes: the Gutenberg-Richter law, the interevent waiting times, the expected time until the next event, the Omori-like and inverse Omori-like behaviour following the example of Osorio et al. (2010). In the analysis of the Gutenberg Richter law of event sizes, we found power-law behaviour with exponents of comparable order (see also Schielicke and Névir, 2011). Compared to tornadoes, the power-law exponent of earthquake magnitudes has a slightly greater value, which could be related to stronger frictional or dissipative processes in the crust of the Earth in comparison to the atmosphere.

In the temporal analyses, we observed different behaviours of earthquakes and tornadoes. While earthquakes show "pure" power-law behaviour, tornadoes generally show power-law behaviour coextensive with characteristic scales. This is especially true when the temporal resolution used for the analysis was high (10 and 60 min bins). In correspondence to the typical tornado diurnal distribution that shows a maximum of tornado occurrences on the late afternoon hours (Schaefer and Edwards, 1999), we found the characteristic timescale to peak near one day in the return times. An explanation might be that the ingredients that are necessary for severe convection (instability, moisture, lift, shear) are more favourably arranged in the afternoon hours (about ingredients-based theory, see e.g. Doswell et al., 1996). We analysed the short time range from about 60 up to $600 \mathrm{~min}$ (mesoscale) and the long time range starting with 1 day (synoptic scale) separately for all tornadoes and for more intense tornadoes with at least $F 2$ Fujita intensities. In the analysis of the interevent waiting times, the short time range (mesoscale) showed power-law behaviour with almost identical exponents of about -1.6 , while the exponents in the synoptic timescale differ: -1.35 for all tornadoes, -2.1 for $F 2+$ tornadoes. Additionally, the pdf estimates of all and $F 2+$ tornado interevent waiting times deviate in the very short time range (less than about $60 \mathrm{~min}$, convective scale). Since the probability for having an $F 2+$ tornado is smaller than that for all tornadoes in this very short time range, it might be a hint that the atmosphere needs slightly more time for rearranging the necessary conditions for the generation of a successive $F 2+$ tornado directly after an $F 2+$ event. The different exponents for tornadoes of different intensity ranges in the larger timescale (larger than one day) reflect the fact that stronger tornadoes $(F 2+)$ are rarer compared to the whole data set including $F 0$ and $F 1$ events. However, it might also be connected to the underlying processes: favourable conditions (arrangement of the necessary ingredients) for producing $F 2+$ tornadoes might be rarer and possibly have different thresholds. Therefore, the return times between two $F 2+$ events are different (longer) which is expressed by a smaller exponent. This is also supported by the analysis of the yearly variations of the exponents of the interevent waiting times of all tornadoes. During the year, the short time exponents also showed only slight variations around -1.6 , while the long time range showed large variations from about -1 in winter to -3.5 in summer. The long time range exponents are connected to the synoptic scale and the synoptic situation. In summer the favourable conditions for producing severe convection and tornadoes are more frequent, and therefore the exponents tend to decrease. In contrast to tornadoes, no characteristic timescales are observed in the earthquake data.

The analysis of the expected time until the next event showed an increase with time for tornadoes and for earthquakes, supporting the thesis that the probability density distributions of tornadoes and earthquakes are heavy-tailed distributions. The curves can be approximated by power laws with different exponents for tornadoes and earthquakes as well as for different intensity classifications (e.g. $F 2+$ versus all tornadoes). The characteristic timescale around multiples of 1 day is reflected by the wave-shaped appearance of the tornado curves. The temporal investigation of the tornadoes support the observation that tornadoes cluster in time. This clustering occurs mainly on the mesoscale, while on the convective times scale, as well as on the synoptic scale, the temporal behaviour is characterised by different exponents. The analysis of Omori and inverse Omori-like behaviour showed a very fast decay of tornado activity following and preceding a main event, while earthquakes show increased seismic activity over a very large time range (days to years, Osorio et al., 2010) in the aftershock sequence. In the case of earthquakes, the observed foreshock sequence is different from the aftershock sequence, since foreshocks are rarer than aftershocks. The difference in the aftershock and foreshock sequence of earthquakes and tornadoes can be attributed to a different timescale of the memory of the material (Earth crust, atmosphere). The atmosphere has a very short memory and is strongly affected by the diurnal variation, while stresses in the Earth's crust can be accumulated over a very large time span.

In summary, for tornadoes, power-law behaviours in the temporal analysis coextensive with characteristic timescales of about one day are observed, while earthquakes show pure power laws. The exponents of the power laws are of the same order for both systems, but uncertainties remain concerning the meaning of their explicit exponent values. Finally, we discussed the applicability of the threshold oscillator concept to tornadoes with the result that it seems to be a plausible concept for the generation of tornadoes requiring more research. The concept supports the assumption that earthquakes and 
tornadoes have a similar underlying structure. The differences between earthquakes and tornadoes can mainly be attributed to the different media the events occur in, but uncertainties remain and pose a challenge for future research on this topic.

Acknowledgements. We would like to thank Christoph Gatzen for helpful and stimulating discussions concerning the paper. We appreciate the helpful and stimulating comments of two anonymous reviewers. This work is part of the PhD-project of Lisa Schielicke, funded by the Helmholtz graduate research school GeoSim. The facilities of the Southern California Earthquake Data Center (SCEDC), and the Southern California Seismic Network (SCSN) were used for access to earthquake data required in this study. The SCEDC and SCSN are funded through US Geological Survey Grant G10AP00091, and the Southern California Earthquake Center, which is funded by NSF Cooperative Agreement EAR-0529922 and USGS Cooperative Agreement 07HQAG0008. The Southern California Seismic Network (SCSN ) catalogue is available online under www.data.scec.org.

Edited by: D. Maraun

Reviewed by: two anonymous referees

\section{References}

Ben-Zion, Y.: Appendix 2, Key Formulas in Earthquake Seismology, in: International Handbook of Earthquake and Engineering Seismology, Part B, edited by: Lee, W. H., Kanamori, H., Jennings, P. C., and Kisslinger, C., Academic Press, 1857-1875, 2003.

Brooks, H. E.: On the Relationship of Tornado Path Length and Width to Intensity, Weather Forecast., 19, 310-319, 2004.

Brooks, H. E. and Doswell III, C. A.: Some aspects of the international climatology of tornadoes by damage classification, Atmos. Res., 56, 191-201, 2001.

Brooks, H. E., Doswell III, C. A., and Kay, M. P.: Climatological estimates of local daily tornado probability, Weather Forecast., 18, 626-640, 2003.

Chakraborti, A. and Patriarca, M.: Variational Principle for the Pareto Power Law, Phys. Rev. Lett., 103, 228701, doi:10.1103/PhysRevLett.103.228701, 2009.

Davis, P. M., Jackson, D. D., and Kagan, Y. Y.: The longer it has been since the last earthquake, the longer the expected time till the next?, Bull. Seism. Soc. Am., 79, 1439-1456, 1989.

Doswell III, C. A. and Bosart, L. F.: Extratropical synoptic-scale processes and severe convection, Meteorol. Monogr., 28, 27-70, 2001.

Doswell III, C. A., Brooks, H. E., and Maddox, R. A.: Flash flood forecasting: An ingredients-based methodology, Weather Forecast., 11, 560-581, 1996.

Doswell III, C. A., Edwards, R., Thompson, R. L., Hart, J. A., and Crosbie, K. C.: A simple and flexible method for ranking severe weather events, Weather Forecast., 21, 939-951, 2006.

Doswell III, C. A., Brooks, H. E., and Dotzek, N.: On the implementation of the enhanced Fujita scale in the USA, Atmos. Res., 93, 554-563, 2009.
Dotzek, N., Grieser, J., and Brooks, H. E.: Statistical modeling of tornado intensity distributions, Atmos. Res., 67-68, 163-187, 2003.

Dotzek, N., Kurgansky, M. V., Grieser, J., Feuerstein, B., and Névir, P.: Observational evidence for exponential tornado intensity distributions over specific kinetic energy, Geophys. Res. Lett., 32, L24813, doi:10.1029/2005GL024583, 2005.

Englehardt, J. D.: Scale invariance of incident size distributions in response to sizes of their causes, Risk Anal., 22, 369-381, 2002.

Feuerstein, B., Dotzek, N., and Grieser, J.: Assessing a tornado climatology from global tornado intensity distributions, J. Climate, 18, 585-596, 2005.

Fujita, T. T.: Proposed characterisation of tornadoes and hurricanes by area and intensity, SMRP research paper no. 91, University of Chicago, 42 pp., 1971.

Godano, C. and Pingue, F.: Is the seismic moment-frequency relation universal?, Geophys. J. Int., 142, 193-198, 2000.

Gutenberg, B. and Richter, C. F.: Earthquake magnitude, intensity and acceleration, Bull Seismol. Soc. Am., 46, 105-145, 1956.

Hainzl, S., Zöller, G., and Scherbaum, F: Earthquake clusters resulting from delayed rupture propagation in finite fault segments, J. Geophys. Res., 108, 2013, doi:10.1029/2001JB000610, 2003.

Herz, A. V. M. and Hopfield, J. J.: Earthquake cycles and neural reverberations: collective oscillations in systems with pulsecoupled threshold elements, Phys. Rev. Lett., 75, 1222-1225, 1995.

Hutton, K., Woessner, J., and Hauksson, E.: Earthquake Monitoring in Southern California for Seventy-Seven Years (1932-2008), Bull. Seismol. Soc. Am., 100, 423-446, 2010.

Jones, L. M. and Molnar, P.: Some characteristics of foreshocks and their possible relationship to earthquake prediction and premonitory slip on faults, J. Geophys. Res., 84, 3596-3608, doi:10.1029/JB084iB07p03596, 1979.

Kagan, Y. and Knopoff, L.: Statistical study of the occurrence of shallow earthquakes, Geophys. J. Astr. Soc., 55, 67-86, 1978.

Kurgansky, M. V.: The statistical distribution of intense moistconvective, spiral vortices in the atmosphere, Doklady Earth Sci., 371, 408-410, in english available at: essl.org/pdf/ Kurgansky2000.pdf, 2000.

Love, T. and Burton, C.: General practice as a complex system: a novel analysis of consultation data, Family Practice, 22, 347352, 2005.

Maeda, K.: Time distribution of immediate foreshocks obtained by a stacking method, Pure Appl. Geophys., 155, 381-394, 1999.

Malamud, B. D. and Turcotte, D. L.: Statistics of severe tornadoes and severe tornado outbreaks, Atmos. Chem. Phys., 12, 84598473, doi:10.5194/acp-12-8459-2012, 2012.

Okal, E. A. and Romanowicz, B. A.: On the variation of bvalues with earthquake size, Phys. Earth Planet. Int., 87, 55-76, doi:10.1016/0031-9201(94)90021-3, 1994.

Omori, F.: On the aftershocks of earthquakes, J. Coll. Sci. Imp. Univ. Tokyo, 7, 111-200, 1894.

Osorio, I., Frei, M. G., Sornette, D., Milton, J., and Lai, Y.: Epileptic seizures: Quakes of the brain?, Phys. Rev. E, 82, 021919, doi:10.1103/PhysRevE.82.021919, 2010.

Pisarenko, V. F. and Sornette, D.: Characterization of the frequency of extreme earthquake events by the generalized Pareto distribution, Pure Appl. Geophys., 160, 2343-2364, 2003. 
Pisarenko, V. F. and Sornette, D.: Rigorous statistical detection and characterization of a deviation from the Gutenberg-Richter distribution above magnitude 8 in subduction zones, Pure Appl. Geophys., 161, 839-864, 2004.

Schaefer, J. T. and Edwards, R.: The SPC tornado/severe thunderstorm database, Preprints, 11th Conf. on Applied Climatology, Dallas, TX, Amer. Meteor. Soc., 10-15 January 1999, 603-606, 1999.

Schielicke, L. and Névir, P.: On the theory of intensity distributions of tornadoes and other low pressure systems, Atmos. Res., 93, 11-20, 2009.

Schielicke, L. and Névir, P.: Introduction of an atmospheric moment combining Eulerian and Lagrangian aspects of vortices: Application to tornadoes, Atmos. Res., 100, 357-365, 2011.
Sornette, D. and Knopoff, L.: The paradox of the expected time until the next earthquake, Bull. Seismol. Soc. Am., 87, 789-798, 1997.

SPC: Storm Prediction Warning Center - Severe Weather Database Files (1950-2006), available at: http://www.spc.noaa.gov/wcm/ \#data (last access: 15 January 2012), 2012.

Stanley, H. E., Amaral, L. A. N, Gopikrishnan, P., Ivanov, P. Ch., Keitt, T. H., and Plerou, V.: Scale invariance and universality: organizing principles in complex systems, Physica A, 281, 60$68,2000$.

Suarez, M. J. and Schopf, P. S.: A Delayed Action Oscillator for ENSO, J. Atmos. Sci., 45, 3283-3287, 1988.

Verbout, S. M., Brooks, H. E., Leslie, L. M., and Schultz, D. M.: Evolution of the US tornado database: 1954-2003, Weather Forecast., 21, 86-93, 2006. 